\title{
A THEOREM ON $C^{*}$-EMBEDDING
}

\author{
F. K. DASHIELL, JR.
}

ABSTRACT. Theorem. In a totally nonmeager and regular space, every countable intersection of open, normal, $C^{*}$-embedded subsets is normal and $C^{*}$-embedded.

A subspace $G$ of a topological space $S$ is called $C^{*}$-embedded if every bounded continuous $f: G \rightarrow \mathbf{R}$ extends continuously to $S$. This note gives a short and elementary proof of the theorem in the abstract. This extends a result of E. Aron ([1], otherwise unpublished) which asserts that in a compact space, every countable intersection of dense, $C^{*}$-embedded, open $F_{\sigma}$ subsets is $C^{*}$-embedded. The original proof in [1] is rather long and nonelementary. The interest of this theorem and its relevance to certain problems in rings of continuous functions are discussed in $[4, \S 6],[5]$, and $[6, \S 5.8]$.

A space is called totally nonmeager (see [2, p. 252]) if every closed subspace is second category (i.e., nonmeager) relative to itself.

Proof of THE THEOREM. Suppose $S$ is a totally nonmeager, regular space and $G_{1}, G_{2}, \ldots$ are open, normal, $C^{*}$-embedded subsets. It suffices to prove disjoint closures in $S$ (for then, since $G_{1}$ is normal and $\bar{Z}_{1} \cap \bar{Z}_{2} \cap G_{1}=\varnothing, G$ would be normal and $C^{*}$-embedded in $G_{1}$ [3, Theorem 1.17]). Put

$$
K=\bar{Z}_{1} \cap \bar{Z}_{2} \text {. }
$$

Clearly $G \cap K=\varnothing$. Suppose (toward a contradiction) that $K \neq \varnothing$. Fix $n$, pick any closed neighborhood $F$ of a point in $K$, and set $A_{i}=G_{n} \cap \bar{Z}_{i} \cap F$, $i=1,2$. Then

$$
\varnothing \neq \overline{Z_{1} \cap F} \cap \overline{Z_{2} \cap F} \subset \overline{A_{1}} \cap \overline{A_{2}},
$$

so the relatively closed subsets $A_{1}, A_{2}$ of the normal, $C^{*}$-embedded set $G_{n}$ do not have disjoint closures. Thus

$$
\varnothing \neq A_{1} \cap A_{2}=G_{n} \cap K \cap F .
$$

Since $F$ was arbitrary and $K$ is regular, $G_{n} \cap K$ is a dense relatively open subset of the nonmeager space $K$ (for all $n$ ). Thus $\varnothing \neq \cap_{n=1}^{\infty}\left(G_{n} \cap K\right)=$ $G \cap K$, a contradiction.

\section{REFERENCES}

1. E. Aron, Embedding lattice-ordered algebras in uniformly closed algebras, Thesis, U. Rochester, 1971.

Received by the editors August 22, 1977.

AMS (MOS) subject classifications (1970). Primary $54 C 45$. 
2. N. Bourbaki, General topology, Part 2, Hermann, Paris; Addison-Wesley, Reading, Mass., 1966.

3. L. Gillman and M. Jerison, Rings of continuous functions, Van Nostrand, Princeton, N. J.; Reinhold, New York, 1960.

4. A. W. Hager, A class of function algebras (and compactifications, and uniform spaces), Symposia Mathematica, Vol. 17, Academic Press, New York, pp. 11-23.

5. A. W. Hager, P. Nanzetta and D. Plank, Inversion in a class of lattice-ordered algebras, Colloq. Math. 24 (1972), 225-234.

6. A. W. Hager and L. C. Robertson, Representing and ringifying a Riesz space, Symposia Mathematica, vol. 21, Academic Press, New York, 1977, pp. 411-431.

Department of Mathematics, California Institute of Technology, Pasadena, CaliFORNIA 91125

Current address: R and D Associates, P. O. Box 9695, Marina del Rey, California 90291 